\section{P158 A REVIEW OF ATTENDANCE AT A WEEKLY PEER-TO- PEER SUPPORT GROUP FOR EXPRESSING MOTHERS IN A PAEDIATRIC HOSPITAL SETTING}

${ }^{1}$ Catherine Carroll, 'Eimear Ryan, ${ }^{2}$ Anne Marie Shine*. 'Our Lady's Children's Hospital, Dublin, Ireland; 'Our Lady's Children's Hospital, Crumlin, Dublin, Ireland

\subsection{6/archdischild-2019-epa.513}

Background A quality improvement project (QIP) was undertaken in 2016 with the goal of achieving 100\% of eligible infants $<2$ weeks of age admitted to Our Lady's Children's Hospital Crumlin (OLCHC) having access to their own mother's milk for the duration of their hospital stay. From this QIP, a weekly peer-to-peer support (PPS) group, facilitated by a dietitian/breastfeeding champion, was established to support expressing mothers in OLCHC. PPS has been shown to be an effective model for increasing breastfeeding rates.

Objectives Establish if the introduction of a 'referral card' for the weekly PPS group would improve attendance.

Methods A retrospective audit of attendance records at a paediatric hospital based PPS group between October 2016 and October 2018. Attendance was compared pre- and postthe introduction of a referral card to the PPS group. Descriptive statistics were undertaken.

Results One hundred meetings occurred during the audit time period with a total of 111 mother contacts. A mean of 1 mother (range $0-4$ ) attended a meeting prior to the introduction of the referral card. Attendance increased to a mean of 2 mothers (range 0-6) after this time $(P=0.0009)$.

Future plans We believe that the establishment of a weekly PPS group, in addition to other measures implemented, has played a role in supporting mothers to continue to express for the duration of their infant's hospital stay. The introduction of the referral card has led to improved attendance at the PPS group. As part of the next PDSA cycle, we hope to survey the mothers who have attended the group so as to collect qualitative data on their experience and gain valuable information on how best we can continue to support these women.

\section{P159 LOW RATE VASCULAR ACCESS RELATED INFECTIONS IN THECHRONIC HAEMODIALYSIS PATIENT COHORT IN A TERTIARY PAEDIATRIC HOSPITAL}

Maria Raftery*, Tara Raftery, Joan Flynn, Karina Murray, Clodagh Sweeney, Malcolm Lewis, Maria Stack, Michael Riordan, Niamh Dolan, Atif Awan. Children's University Hospital, Temple Street, Dublin, Ireland

\subsection{6/archdischild-2019-epa.514}

Background Vascular access is required for haemodialysis (HD), a form of renal replacement therapy required when a child has acute or end stage kidney disease (ESKD). Central venous catheter (CVC) line infections are the second most common cause of vascular access loss in the long term HD patient, commonly caused by poor hand hygiene practices, clinical environment or inadequate sterilisation procedures. In the literature the reported rates of infection range from $0.8-4.8$ episodes $/ 1000$ catheter days in this patient group.
Aim To audit CVC line related infection rates in our tertiary HD unit in Temple Street Children's University Hospital (TSCUH) over a 5 year period.

Methods A retrospective review was carried out on all patients who had HD from January 2012- January 2017 (inclusive) using a CVC.

Results/Findings 34 patients required HD, of which 44\% were female and $56 \%$ male.

Twenty-three patients (68\%) were aged between 5-10 years of age.

Dysplastic kidneys was the most common reason for requiring HD $(n=9,26.2 \%)$. Eight patients required HD for 1-6 months, 5 patients for 6-12 months, 19 patients for 12-48 months, and 2 patients for $>60$ months.

Five out of 34 children developed CVC line infections with an overall infection rate of 0.22 episodes per 1000 catheter days. Loss of protective caps was the main reason for infection $(n=3)$. Staph-aureous, pseudomonas oryzihabitans, enterococcus faecalis and mixed coagulase negative staphylococci were the identified organisms causing infection.

Conclusions CVC line infections in long-term HD patients attending TSCUH are maintained at a low rate in comparison to published data.

Strict application of Aseptic Non Touch Technique shows favourable results without the need for prophylactic antibiotics locks. Future work includes a cost benefit analysis of this practice.

\section{P160 SAFETY CLIMATE SURVEY - ARE WE GETTING BETTER?}

Sanjeev Kamath*, Ahmed Khan. Daisy Hill Hospital, SHSCT, Newry, UK

\subsection{6/archdischild-2019-epa.515}

Background Organisations working towards a culture of safety need a reliable measure to monitor the success of their initiatives. A Safety Climate Survey was carried out during September 2017 in the Paediatric ward at Daisy Hill Hospital, as part of the S.A.F.E. (Safety Awareness for Everyone) initiative. A number of quality and safety measures were undertaken over the last 18 months.

Aim To repeat the Safety Climate Survey and gauge safety culture in the department of Paediatrics at Daisy Hill Hospital (DHH).

Method

- RCPCH - Safety Climate Survey was used. This was previously used in 2017.

- Survey was completed by multi-Disciplinary staff including all grades of medical \& nursing team in the Paediatric department, DHH. Administrative support staff, medical students \& health care assistants were also included.

- An email was sent to all staff at the beginning of the survey.

- Hard copies of survey sheets were given to staff over a period of 3 weeks during January 2019 with a central collection point in the children's ward. A previous attempt in 2017 of digital surveys had a poor response.

- Survey data analysed and compared with previous (2017) results.

Results

- Fifty (69\%) completed survey forms received from a staff pool of 72 . 
- Following table provides summary \& comparative data:

\begin{tabular}{llll}
\hline & Survey Domains & 2017 & $\mathbf{2 0 1 9}$ \\
\hline a & Staff in this ward take responsibility for patient safety & $89 \%$ & $94 \%$ \\
b & Briefing staff on handovers is good for patient safety & $74 \%$ & $94 \%$ \\
c & Happy with Clinical Leadership - Medical & $71 \%$ & $74 \%$ \\
d & Happy with Clinical Leadership - Nursing & $68 \%$ & $72 \%$ \\
e & Encouraged by colleagues to report safety concerns & $68 \%$ & $88 \%$ \\
f & Medical \& Nursing leaders listen \& care about my & $60 \%$ & $68 \%$ \\
& concerns & & \\
g & Leadership drive to a safety centered organisation & $58 \%$ & $66 \%$ \\
h & More patient safety now than a year ago & $55 \%$ & $60 \%$ \\
i & Senior leaders listen \& care about my concerns & $50 \%$ & $52 \%$ \\
j & Difficult to discuss errors at work & $18 \%$ & $24 \%$ \\
\hline
\end{tabular}

- Results will be shared with staff

Conclusion

- Safety climate survey can provide greater understanding of the organisational safety culture.

- Improvements have been noticed in all domains. These results reiterated the prevailing safety culture in the department.

- We plan to implement Senior Safety Walks and Safety Huddles to further improve openness.

\section{REFERENCES}

1. RCPCH Safety Survey; https://www.rcpch.ac.uk/system/files/protected/page/2a\% 20\%20Safety\%20Climate\%20Survey.pdf

2. http://primaris.org/sites/default/files/resources/Patient $\% 20$ Safety/safety\%20climate $\% 20$ survey.pdf

3. http://www.health.org.uk/publication/evidence-how-safe-are-clinical-systems

\section{P161 PAEDIATRIC ANALGESIA: PRESCRIBING PATTERNS AND CHALLENGES FACING SURGICAL NCHDS IN A NON- SPECIALIST PAEDIATRIC HOSPITAL}

Mai O'Sullivan*, Anne Dolan. Sligo University Hospital, Sligo, Ireland

\subsection{6/archdischild-2019-epa.516}

Introduction Acute pain is one of the most common adverse stimuli experienced by children, often recurring pre and postoperatively. It is associated with increased anxiety, somatic symptoms, avoidance and increased parental distress ${ }^{1}$. Effective treatment is essential to prevent long term psychological changes $^{2}$. An Acute Pain Service (APS) was established in Sligo University Hospital in January 2019 to meet the growing demands of our peri-operative inpatient population. At this time, little was known about the current local practices of our surgical colleagues when prescribing simple analgesia for children.

Aims This study aimed to investigate the prescribing patterns and challenges faced by Surgical Non-Consultant Hospital Doctors (NCHDs) when prescribing for children.

Methods A paper-based survey was conducted in January 2019. It was sent to all General Surgical, Orthopaedic and Ear Nose and Throat NCHDs. It contained 24 questions. They related to teaching received in paediatric prescribing, familiarity with pain assessment tools used to quantify children's pain, including the FACES score and the sources of information currently used to calculate doses. We asked specifically about prescribing for children in the obese and underweight categories. Confidence levels prescribing analgesia for children was assessed using a Likert Scale. The involvement of parents and nursing staff in the management of pain by surgical NCHDs was also assessed.

Results The paper-based survey was administered to 32 NCHDs. Experience level ranged from Intern to Specialist Registrar. The response rate was 68\%. The majority of respondents did not feel confident prescribing analgesia for children. The BNF for children was the most common source used for dosing calculations. 12\% selected the correct method for prescribing analgesia for the obese and underweight child. There was poor involvement of parents and nursing staff in analgesia planning and engagement in distraction techniques.

Conclusion Surgical NCHDs in our institution are uncomfortable prescribing simple analgesia for children. They are open to, and would likely benefit from receiving educational sessions on this topic from the APS. Further guidance will be offered to surgical NCHDs in our institution going forward, particularly in relation to children who are at extremes of weight.

\section{REFERENCES}

1. American Academy of Paediatrics, American Pain Society. The Assessment and Management of Acute Pain in Infants, Children and Adolescents. Paediatrics 2001; 108(3):793-797.

2. Kulshrestha, A. and Bajwa, S. (2019). Management of acute postoperative pain in pediatric patients. Anaesthesia, Pain \& Intensive Care, 22, 3(1607-8322), pp.2220-5799.

\section{P162 USING IMPLEMENTATION SCIENCE METHODOLOGY TO ACHIEVE QUALITY IMPROVEMENT OUTCOMES FOR THE NATIONAL HEALTHY CHILDHOOD PROGRAMME IN IRELAND}

Phil Jennings*, Fiona McGuire, Carmel Brennan, Anne Pardy. Department of Public Health HSE, Tullamore, Ireland

10.1136/archdischild-2019-epa.517

On average over 62,000 babies are born in Ireland every year. Life expectancy for men and women in Ireland has increased significantly since the foundation of the state. There is good evidence that investment in early childhood is costeffective, delivering both an economic and a social return. One of the ways to assure these returns is to deliver a universal, evidence-based child health programme, thereby achieving the best outcomes for children.

Ireland's 'National Healthy Childhood Programme' is free to all children up to the age of 14 years. In common with international models, it consists of three components: child health assessments, screening and immunisations. In 2014 the Health Service Executive's Child Public Health Group reviewed the international evidence and updated the existing child health programme (Best Health for Children 2005). This also triggered the development of formal structures to support child health screening, a restructuring of some of the key child health contacts and the implementation of an ambitious programme of work called the 'Nurture Programme - Infant Health and Wellbeing'. The focus of Nurture was to improve the knowledge and professional skills of front-line providers of the service and also the information and support available to parents during pregnancy and for the first three years of their child's life. 